\title{
Moving balance and mobility evidence in to action: A primer in Knowledge Translation
}

KM Sibley, $\mathrm{PhD}^{1,2}$, SE Straus, MD, MSc ${ }^{3}, \mathrm{~F} \mathrm{Webster}^{4}$, SB Jaglal, $\mathrm{PhD}^{1,2}$

${ }^{1}$ Toronto Rehabilitation Institute; ${ }^{2}$ Department of Physical Therapy, University of

Toronto; ${ }^{3}$ Li-Ka-Shing Knowledge Institute, St. Michael's Hospital; ${ }^{4}$ Departments of

Surgery and Health Policy, Management and Evaluation, University of Toronto; Toronto,

Ontario, Canada

\section{CORRESPONDING AUTHOR}

Dr SB Jaglal, 500 University Avenue Rm 160, Toronto, Ontario, Canada M5G 1V7, 416

978-0315, susan.jaglal@ utoronto.ca

\section{ACKNOWLEDGEMENTS}

We thank Dr. G. Mochizuki for his helpful comments regarding early versions of the manuscript. KM Sibley is supported by a Fellowship from the Canadian Institutes of Health Research and the Toronto Rehabilitation Institute. SE Straus holds a Canada Research Chair in Knowledge Translation. SB Jaglal holds the Toronto Rehabilitation Institute Chair in Rehabilitation Research at the University of Toronto. We acknowledge the support of the Toronto Rehabilitation Institute who receives funding under the Provincial Rehabilitation Research Program from the Ministry of Health and Long Term Care in Ontario.

\section{KEYWORDS}

balance, mobility, knowledge translation, review, knowledge to action 


\begin{abstract}
Knowledge generated by research into the control of posture and gait has many implications for individuals with balance and mobility impairments. Communicating such knowledge through conference presentations and peer-reviewed manuscripts is appropriate for some audiences, but does not effectively reach all potential knowledge users (for example: clinicians, policy-makers, and the public). Expanding interest in promoting evidence-based decision-making and practice to ensure that research results reach the target end-user has lead to the emergence of the field of Knowledge Translation (KT). The focus of KT is to improve the dissemination and uptake of knowledge in decision-making, and is grounded in a number of theoretical frameworks and its own rapidly expanding literature base. As many general principles of KT can be applied to the optimization of balance and mobility, the purpose of this review is to serve as a primer for gait and posture researchers. Two major models of KT are summarized: the National Institutes of Health (NIH) Roadmap and Knowledge-to-Action Framework. The principles outlined in these frameworks are applied to guidelines to assist researchers with developing their own evidence-based KT strategies. Examples of balance and mobility-specific KT activities are provided when appropriate, but there is a need for more studies that attempt to use evidence-based KT strategies to change practice in this particular field.
\end{abstract}




\section{Introduction}

Research in the control of posture and gait integrates basic and applied science in the context of maximizing mobility for people across the continuum of functional abilities. The balance and mobility research community produces a high volume of knowledge (28,589 Pubmed hits for 'postural control' and 'gait' on May 6, 2010), ranging from experiments determining the fundamental mechanisms controlling posture and gait, to evaluations of interventions for improving functional abilities in patient populations. However, like other domains of health science, the best evidence for promoting balance and mobility is not always incorporated into clinical practice. For example, through a consensus discussion and survey, the Gait and Clinical Movement Analysis Society recognized that gait analysis is inconsistently used in clinical decision making due in part to a lack of basic understanding of the benefits and limitations of gait and motion analysis among clinicians [1]; and in a qualitative study of clinical decision making, physical therapists reported using informal observation as their primary diagnostic tool for balance assessment despite available standardized measures [2].

These examples suggest the presence of a gap between the evidence produced by the balance and mobility research community, and what is done in the 'real world'. Accordingly, there is a need for systematic processes to ensure that the best evidence reaches the appropriate end-users- whether they may be clinicians, health administrators, other researchers or policy makers. With its aim to improve the uptake of knowledge in decision-making, Knowledge Translation (KT) is a rapidly growing field that has implications for all knowledge users (anyone with an interest in or use of research results). KT is a science with its own evidence base and emerging understanding of the 
most effective tools to move knowledge into action. Its generalized principles can be applied across multiple clinical settings- including the field of gait and balance.

No single individual or group is responsible for the entire process of knowledge generation and translation [3], however, clinical researchers are in a unique position to facilitate moving research evidence into practice. Funding agencies are recognizing this role and are increasingly requiring applicants to demonstrate planned, systematic approaches to disseminating research findings- both within and beyond the scientific community [4]. However, as these principles have not traditionally been a component of academic training [3], there may be a gap in KT knowledge and evidence reaching researchers who may benefit from applying it to their own work. As the journal Gait and Posture publishes a high proportion of clinical research articles related to patient populations with balance and mobility impairments, this paper attempts to bridge some gaps by bringing a brief overview of KT to readers who may undertake translational activities in their specific fields related to optimizing balance and mobility. This review can serve as a primer in $\mathrm{KT}$, the purpose of which is to review the basic principles and framework for how to 'do’ KT for application in balance and mobility research.

\section{Defining KT}

A recent study identified over 100 terms relating to knowledge translation [5], including knowledge transfer, exchange, utilization and implementation [6]. While such variety in terminology can lead to confusion with respect to both defining a common lexicon and accessing relevant information, the most commonly cited definition of KT originates from Canada and has been adopted by several international agencies $[7,8]$ : "a dynamic and iterative process that includes synthesis, dissemination, exchange and 
ethically-sound application of knowledge to improve the health of Canadians, provide more effective health services and products and strengthen the health care system" [9]. This operational definition encompasses the steps necessary to move knowledge into action. $\mathrm{KT}$ is broader in scope than technology transfer and continuing education, and its audience includes various decision makers such as clinicians, patients, researchers and policy makers [10]. Despite the variation in KT terminology, common to all definitions is the concept of moving beyond simple dissemination of knowledge to actual use[10].

\section{Models of KT}

The use of theories to understand KT provides a conceptual framework for studying research utilization. Many knowledge translation theories exist, based in cognitive psychology, planned change, education, organizational theory and quality improvement [11]. In this review we highlight two major KT frameworks, and readers can refer to Estabrooks et al. [12] and Sudsawad [13] for summaries of other KT models.

\section{The NIH Roadmap}

The National Institutes of Health (NIH) Roadmap [14] identified two major blocks in the translation of research findings into practice: T1- related to the transfer of understanding of disease mechanisms into development of new diagnosis techniques and therapies; and T2- translation of the results of clinical studies into every day decisionmaking and practice [15]. A revised Roadmap was later proposed which divided the T2 block into 'practice-based research' to translate findings to patients (such as guideline preparation and systematic reviews) and T3:'translational research' to translate understanding to clinical settings and address problems encountered by primary healthcare professionals using implementation research (Figure 1) [16]. This T3 
component emphasizes the importance of 1) identifying challenges with incorporating best practice into clinical care, 2) demonstrating the sustainability of interventions in the 'real-world', and 3) evaluating system improvements [16].

\section{Knowledge to Action (KTA)}

The KTA framework (Figure 2) [6] encompasses two phases: the knowledge creation phase and the action cycle. The knowledge creation phase funnels the large body of discovery research (including basis and clinical science) that encompasses knowledge inquiry into knowledge syntheses with systematic reviews and meta-analyses, and ultimately forms knowledge products such as best practice guidelines. The goal is that knowledge becomes more useful to the end-user as it is funneled through this refinery. The action cycle identifies the steps required for a given unit of knowledge to reach widespread use. First, a gap must be identified between current practice and the evidence. Subsequently, to address the identified gap, the evidence must be adapted to the local environment while simultaneously addressing relevant barriers and facilitators to using the evidence in that particular situation. Strategic interventions (targeted to the barriers and facilitators) are required to facilitate uptake of the knowledge, followed by ongoing monitoring and evaluation to assess the impact of knowledge use and ensure knowledge use is sustained.

Both frameworks highlight the identification of gaps in knowledge use relative to the evidence base as a critical point for further attention and intervention. The NIH Roadmap focuses on where gaps exist and what needs to be done to fill them, while the KTA framework provides actionable steps for identifying and filling such gaps based on an 
iterative process. Building on these frameworks, in the following section we provide researchers with suggestions to adapt these tools to their own work.

\section{How to 'do' Knowledge Translation}

The first step in any KT endeavor is to identify the purpose for translating a piece of evidence. In general, two major goals of knowledge translation are 1) to communicate knowledge and 2) to change behavior. If the goal is to communicate results to other researchers, diffusion strategies such as conference presentations and peer-reviewed manuscripts are appropriate [17]. If communicating to groups such as clinicians, health administrators, policy makers or the general public, dissemination strategies that tailor the message and delivery medium to the target audience are more effective [17]. This requires customizing the message for the audience, identifying a credible individual(s) to deliver the message, and considering what factors might influence receiving the message [18]. Dissemination strategies include user workshops, networks of practice, use of opinion leaders, and media engagement. Networks or communities of practice bring together individuals with a common interest to fulfill goals typically related to improving professional practice [19]. A well-known example is the ProFaNE (Prevention of Falls Network Europe) group, with over 4000 members[20]. This group coordinates research endeavors such as clinical trials, as well as dissemination activities, such as coordinating a comprehensive website that shares research results and organizing workshops.

While dissemination strategies foster collaboration and associations between members, there is little evidence of their effectiveness at actually changing practice. When the KT goal is to change some behavior among end users, a systematic approach is required that examines the factors associated with changing the behavior and develops an 
intervention target to address these factors and using the best implementation evidence. Translating knowledge to change behavior comprises four key phases: 1) evaluating the evidence; 2) identifying evidence-practice gaps; 3) developing an intervention to change practice and outcomes; and 4) evaluating the intervention. These steps are described in the following sections.

\section{Evaluating the level of evidence}

Prior to launching any KT intervention, it is necessary to critically examine whether there is sufficient evidence to support changing behavior on a broad scale. If the body of evidence is small (only one or two small trials) or of low quality, the knowledge may not be ready for widespread dissemination. There may be a need to conduct additional studies to strengthen the evidence base. The systematic review [21] is the basic tool for evaluating the state of a science when considering whether a change in practice is justified. The conclusions of the systematic review therefore direct the KT intervention itself. For example, systematic reviews for fall prevention have identified which interventions are most efficacious (multi-component exercise) and which are less so (home hazard modification and vitamin D supplementation) at preventing falls in community-dwelling elderly[22]. It is also important to note the limitations of systematic reviews. For example, the nature of data pooling can make it difficult to compare across studies, and important distinctions between interventions may be lost when collapsing results [23]. Accordingly, systematic reviews can provide general information about efficacy, but it may be difficult to replicate study procedures and findings (for example, prescribing specific exercises for fall prevention).

\section{Identifying evidence-practice gaps}


If the review of evidence shows that there is a clear 'best practice', then it is appropriate to determine whether there are any gaps between what is currently practiced and the identified best practice. Such gaps may be identified by researchers or end-users, though it is advantageous if an end-user identifies a gap, because it may suggest a potential willingness to change [24]. While gaps may be identified informally, objective assessments are most useful, as it is possible that 'we don't know what we don't know' [25]. Identifying an evidence-practice gap could include baseline studies examining the prevalence of the issue, environmental scans, needs assessments, examination of administrative data or surveys to document current practice [26]. For example, Bayley et al.[27] identified research and knowledge translation priorities for stroke rehabilitation through expert panel consultation using a modified Delphi voting approach.

\section{Developing an intervention to change practice}

The development of an evidence-based intervention that will change behavior requires planning of multiple components. Firstly, the barriers and facilitators to knowledge must be identified, which may be done through surveys, qualitative interviews or literature reviews [28, 29]. Individual factors like education, attitudes and beliefs, interest, perceived role, and self-efficacy all influence willingness to change [30, 31]. Organizational readiness for change is an important albeit complex issue; dependent on many components such as whether members value change, whether task demands and resources available are feasible, and contextual issues such as a positive climate and positive past experiences with change[32]. An ongoing focus in KT science is the assessment of organizational readiness for change, and preliminary data on such tools suggests that these factors do play a role in implementation success and can be predicted 
$[33,34]$. Second, once the determinants of knowledge use have been identified, it is necessary to adapt the evidence to the local context. Adaptation is necessary because different entities may already be performing certain elements of best practice or some elements of the evidence may not be feasible in all settings. Formalized tools are available for adapting knowledge that has been integrated into best practice guidelines $[35,36]$. These tools outline the elements required for adaptation, including appropriate selection of a panel, identifying what questions to ask and how to ask them, evaluating the evidence with respect to the quality and consistency of the evidence as well as its applicability to the local context [29].

All of the above elements contribute to the selection and design of the KT intervention. There is a growing generalized evidence base on which types of KT interventions are most effective at changing behavior, although there is a need for more evaluation of interventions specific to balance and mobility. Common KT approaches include educational interventions, linkage and exchange interventions, audit and feedback interventions, informatics, and patient-oriented interventions (see Straus et al. for review[37]). A systematic review by Grimshaw et al. [38] determined that most interventions improved care to some degree, but the magnitude of effects varied. They determined that effective options for targeting healthcare providers included reminder systems (checking data against clinical rules and providing prompts for action) [39], outreach visits (taking the intervention to the workplace of the end-user) [40], and to a lesser extent, patient-directed interventions and audit and feedback techniques (providing summaries of health care performance over a given time period) [41]. Multi-faceted interventions were not more effective than single interventions, nor did effectiveness 
increase with the number of interventions [38]. Despite the options, there were concerns that only the 'simplest' interventions (distributing materials and lunchtime sessions) were the only feasible approaches in many environments [38]. A potential limitation of many KT interventions is that they are focused on physicians, and there is limited data for what works among other health professionals, such as physiotherapists [42]. Furthermore, as with other disciplines, the literature on $\mathrm{KT}$ interventions may be subject to a publication bias. Interventions that were not developed or evaluated systematically may not be considered suitable for publication, and as such there may be more examples of both successful and unsuccessful approaches that are not documented.

\section{Evaluating the intervention}

Evaluation of the KT intervention is critical in order to determine whether the intervention caused a change in knowledge use and in relevant clinical or health systems outcomes. Although randomized controlled trials are typically considered to be optimal when evaluating efficacy, they are not always feasible and other designs could be considered (such as interrupted time series). When considering how to evaluate KT interventions, one needs to consider whether the goal is to evaluate knowledge use or the impact of knowledge use [43]. Moreover, it is important to consider that knowledge use

could occur at a conceptual level (a change in understanding or attitudes), an instrumental level (a change in behavior) or persuasive level [6], and that outcomes could be at a decision-maker, patient, or systemic level [44].

\section{Limitations and Future Directions}

The evidence provided by KT science can be applied by clinical researchers across many disciplines, including balance and mobility, but it is an emerging field that is not 
without limitations and opportunities for further development. The many components of a $\mathrm{KT}$ intervention outlined in this paper require a considerable investment of time, invariably forming a research program in itself over the course of several years as each step may require single or multiple studies before moving forward. The tradeoff for the lengthy time requirement is the increased probability of success relative to 'just doing something'.

We have noted that much of the KT literature refers to generalized principles for promoting change, but the subject of the interventions themselves is very specific. There is a need, particularly in the fields of balance and mobility, for more examples of successful and unsuccessful implementation projects, and to expand beyond physicianspecific interventions as the primary agent of change. This is necessary to inform other users and optimize the efficiency of intervention efforts.

KT science is an evolving field and there is also a need for continued development in a number of areas. For example, despite the role of many governments in healthcare funding, we know relatively little about evaluating readiness for change in the political environment. As noted, there is an ongoing need to refine understanding of the effectiveness of KT interventions, and in particular there is a need to determine how to best make many of these practice changes sustainable over the long term.

\section{Conclusions}

Effective knowledge translation requires careful consideration and planning. Moreover, effective KT is not a one-size-fits-all strategy. Context and fit are crucial, as a successful approach in one environment may not extend to other scenarios. There is ongoing debate about who is 'responsible' for knowledge translation; should it be the 
responsibility of researchers, as the producers of knowledge or should the responsibility lie with the users - clinicians, policy makers and the general public? The reality is that both producers and users of knowledge need to be actively engaged and cooperate to determine the best way to apply knowledge to its potential. Thus, knowledge translation is the responsibility of all, not some, and as we better learn how to best work together and integrate with one another, new opportunities and applications for knowledge not previously conceived of are likely to emerge. 


\section{References}

1. Engsberg JR, Tucker C, Ounpuu S, Wren TA, Sisto SA, Kaufman KR. Gait and clinical movement analysis research priorities: 2007 Update from the research committee of the gait and clinical movement analysis society. Gait \& Posture 2009. 29(2): 169-71.

2. McGinnis PQ, Hack LM, Nixon-Cave K, Michlovitz SL. Factors That Influence the Clinical Decision Making of Physical Therapists in Choosing a Balance Assessment Approach. Phys Ther 2009. 89(3): 233-47.

3. Lane J, Flagg J. Translating three states of knowledge--discovery, invention, and innovation. Implementation Science 2010. 5(1): 9.

4. Graham ID, Tetroe J. Getting Evidence into Policy and Practice: Perspective of a Health Research Funder. J Can Acad Child Adolesc Psychiatry 2009. 18(1): 4650 .

5. McKibbon KA, Lokker C, Wilczynski N, Ciliska D, Dobbins M, Davis D, Haynes $\mathrm{RB}$, Straus S. A cross-sectional study of the number and frequency of terms used to refer to knowledge translation in a body of health literature in 2006: a Tower of Babel? Implementation Science 2010. 5(1): 16.

6. Graham ID, Logan J, Harrison MB, Straus SE, Tetroe J, Caswell W, Robinson N. Lost in knowledge translation: Time for a map? J Contin Educ Health Prof 2006. 26(1): 13-24.

7. National Center for the Dissemination of Disability Research, What is Knowledge Translation? FOCUS Technical Brief. 2005, Austin, TX.

8. World Health Organization. Bridging the "Know-Do" Gap Meeting on Knowledge Translation in Global Health. 2005. Geneva Switzerland.

9. Canadian Institutes of Health Research. About Knowledge Translation. [cited; Available from: http://www.cihr-irsc.gc.ca/e/29418.html

10. Straus SE, Tetroe J, Graham I. Defining knowledge translation. CMAJ 2009. 181(3-4): 165-68.

11. Graham ID, Tetroe J, KT Theories Group, Planned-Action Theories, in Knowledge Translation in Health Care: Moving from Evidence to Practice, S. Straus, J. Tetroe, and I. Graham, Editors. 2009, Blackwell Publishing: West Sussex, UK.

12. Estabrooks CA, Thompson DS, Lovely JJE, Hofmeyer A. A guide to knowledge translation theory. J Contin Educ Health Prof 2006. 26(1): 25-36.

13. Sudsawad P, Knowledge translation: Introduction to models, strategies, and measures. 2007, Austin, TX: Southwest Educational Development Laboratory, National Center for the Dissemination of Disability Research.

14. Zerhouni E. MEDICINE: The NIH Roadmap. Science 2003. 302(5642): 63-72.

15. Sung NS, Crowley WF, Jr., Genel M, Salber P, Sandy L, Sherwood LM, Johnson SB, Catanese V, Tilson H, Getz K, Larson EL, Scheinberg D, Reece EA, Slavkin H, Dobs A, Grebb J, Martinez RA, Korn A, Rimoin D. Central Challenges Facing the National Clinical Research Enterprise. JAMA 2003. 289(10): 1278-87.

16. Westfall JM, Mold J, Fagnan L. Practice-Based Research--"Blue Highways" on the NIH Roadmap. JAMA 2007. 297(4): 403-06. 
17. Lomas J. Diffusion, Dissemination, and Implementation: Who Should Do What? Ann N Y Acad Sci 1993. 703(Doing More Good than Harm The Evaluation of Health Care Interventions): 226-37.

18. Tetroe J. What does it mean to transform knowledge into action? in Canadian Falls Prevention Conference: Transforming Knowledge to Action. 2010. Vancouver, BC.

19. Wenger E, McDermott R, Snyder WM, Cultivating Communities of Practice. 2002, Boston, MA: Harvard Business School Press.

20. About ProFaNE. [cited 2010 November 14, 2010]; Available from: http://www.profane.eu.org/about/about.php?sid=bbc793d38088f312e8af40323d1 $\underline{37 d 46 .}$.

21. Tetzlaff J, Tricco AC, Moher D, Knowledge Synthesis, in Knowledge Translation in Healthcare: Moving from Evidence to Practice, S. Straus, J. Tetroe, and I. Graham, Editors. 2009, Blackwell Publishing: West Sussex, UK.

22. Gillespie LD, Robertson MC, Gillespie WJ, Lamb SE, Gates S, Cumming RG, Rowe BH. Interventions for preventing falls in older people living in the community. Cochrane Database of Systematic Reviews 2009(2): Art. No: CD007146.

23. Shepperd S, Lewin S, Straus S, Clarke M, Eccles MP, Fitzpatrick R, Wong G, Sheikh A. Can We Systematically Review Studies That Evaluate Complex Interventions? PLoS Med 2009. 6(8): e1000086.

24. Weiner BJ, Amick H, Lee S-YD. Review: Conceptualization and Measurement of Organizational Readiness for Change: A Review of the Literature in Health Services Research and Other Fields. Med Care Res Rev 2008. 65(4): 379-436.

25. Brown CJ, Gottschalk M, Van Ness PH, Fortinsky RH, Tinetti ME. Changes in Physical Therapy Providers' Use of Fall Prevention Strategies Following a Multicomponent Behavioral Change Intervention. Phys Ther 2005. 85(5): 394403.

26. Kitson A, Straus SE, Identifying the knowledge-to-action gaps, in Knowledge Translation in Healthcare: Moving from Evidence to Practice, S. Straus, J.

Tetroe, and I. Graham, Editors. 2009: West Sussex, UK.

27. Bayley MT, Hurdowar A, Teasell R, Wood-Dauphinee S, Korner-Bitensky N, Richards CL, Harrison M, Jutai JW. Priorities for Stroke Rehabilitation and Research: Results of a 2003 Canadian Stroke Network Consensus Conference. Arch Phys Med Rehabil 2007. 88(4): 526-28.

28. Cabana MD, Rand CS, Powe NR, Wu AW, Wilson MH, Abboud P-AC, Rubin HR. Why Don't Physicians Follow Clinical Practice Guidelines?: A Framework for Improvement. JAMA 1999. 282(15): 1458-65.

29. Harrison MB, Legare F, Graham ID, Fervers B. Adapting clinical practice guidelines to local context and assessing barriers to their use. Can Med Assoc J 2010. 182(2): E078-E84.

30. Salbach NM, Jaglal SB, Korner-Bitensky N, Rappolt S, Davis D. Practitioner and Organizational Barriers to Evidence-based Practice of Physical Therapists for People With Stroke. Phys Ther 2007. 87(10): 1284-303. 
31. Bulley C, Donaghy M, Dow C. Are physiotherapy guidelines for the management of osteoporosis being implemented? A UK-wide survey. Advances in Physiotherapy 2008. 10: 95-103.

32. Weiner B. A theory of organizational readiness for change. Implementation Science 2009. 4(1): 67.

33. Hagedorn H, Heideman P. The relationship between baseline Organizational Readiness to Change Assessment subscale scores and implementation of hepatitis prevention services in substance use disorders treatment clinics: a case study. Implementation Science 2010. 5(1): 46.

34. Helfrich C, Li Y-F, Sharp N, Sales A. Organizational readiness to change assessment (ORCA): Development of an instrument based on the Promoting Action on Research in Health Services (PARIHS) framework. Implementation Science 2009. 4(1): 38.

35. MacDermid J, Brooks D, Solway S, Switzer-McIntyre S, Brosseau L, Graham I. Reliability and validity of the AGREE instrument used by physical therapists in assessment of clinical practice guidelines. BMC Health Services Research 2005. 5(1): 18 .

36. Development and validation of an international appraisal instrument for assessing the quality of clinical practice guidelines: the AGREE project. Quality and Safety in Health Care 2003. 12(1): 18-23.

37. Straus S, Tetroe J, Graham ID, eds. Knowledge Translation in Health Care Moving from Evidence to Practice. 2009, Wiley-Blackwell BMJ Books: West Sussex, UK.

38. Grimshaw JM, Thomas RE, MacLennan G, Fraser C, Ramsay CR, Vale L, Whitty P, Eccles MP, Matowe L, Shirran L, Wensing M, Dijkstra R, Donaldson C. Effectiveness and efficiency of guideline dissemination and implementation strategies. . Health Technol Assess Rep 2004. 8(6).

39. Dexheimer JW, Sanders DL, Rosenbloom ST, Aronsky D. Prompting Clinicians: A Systematic Review of Preventative Care Reminders. AMIA Annu Symp Proc 2005: 938.

40. O'Brien MA, Rogers S, Jamtvedt G, Oxman A, Odgaard-Jensen J, Kristofferson DT, Forsetlund L, Bainbridge D, Freemantle N, Davis D, Haynes RB, Harvey E. Educational outreach visits: effects on professional practice and health care outcomes. Cochrane Database of Systematic Reviews 2007(4):

DOI:10.1002/14651858.CD000409.pub2.

41. Jamtvedt G, Young JM, Kristofferson DT, O'Brien MA, Oxman AD. Audit and feedback: effects on professional practice and health care outcomes. Cochrane Database of Systematic Reviews 2006(2): Art. No.: CD000259. DOI: 10.1002/14651858.CD000259.pub2.

42. Menon A, Korner-Bitensky N, Kastner M, McKibbon KA, Straus SE. Strategies for rehabilitation professionals to move evidence-based knowledge into practice: a systematic review. Journal of Rehabilitation Medicine 2009. 41: 1024-32.

43. Straus SE, Tetroe J, Graham ID, Zwarenstein M, Bhattacharyya O, Monitoring knowledge use and evaluating outcomes of knowledge use, in Knowledge Translation in Health Care: Moving from Evidence to Practice, S. Straus, J. Tetroe, and I. Graham, Editors. 2009, Blackwell Publishing: West Sussex, UK. 
44. Hakkennes $\mathrm{S}$, Green $\mathrm{S}$. Measures for assessing practice change in medical practitioners. Implementation Science 2006. 1(1): 29. 